\title{
An Ethical Issue After the Nuclear Accident in Fukushima: Young People'S Perspectives of Thyroid Cancer Screening and its Harms
}

Sanae Midorikawa ( $\sim$ s-midori@mgu.ac.jp )

Miyagi Gakuin Women's University

Akira Ohtsuru

Nagasaki University

\section{Research Article}

Keywords: Overdiagnosis, thyroid cancer, decision-making, screening, nuclear accident

Posted Date: November 30th, 2021

DOI: https://doi.org/10.21203/rs.3.rs-1081158/v1

License: (c) (1) This work is licensed under a Creative Commons Attribution 4.0 International License.

Read Full License 


\section{Abstract \\ Background}

Overdiagnosis of thyroid cancer has become a major global medical issue. Ultrasound-based thyroid cancer screening has promoted overdiagnosis, and recently international recommendations indicate that such screening should not be conducted, even after a nuclear accident. The Fukushima thyroid cancer screening program was initiated in 2011 as a health policy after the nuclear accident, although the risk for radiation-induced thyroid cancer was unlikely given the low radiation levels. However, the thyroid cancer screening program has continued at 2-year intervals with a relatively high participation rate and is now in its fifth round. Therefore, it is crucial to clarify whether those targeted for screening understand the disadvantages of screening and identify factors that influenced their decision to participate.

\section{Methods}

We conducted an anonymous mail-based questionnaire that included young people from Fukushima (subjects) and a neighboring prefecture that was not targeted for screening (non-subjects). We asked them about the significance of the thyroid cancer screening in Fukushima, the reasons for taking or not taking a screening, their perception of the harms of screening, and their opinions on thyroid examination at school. Then we compared the results of the questionnaire between subjects and non-subjects and examinees (who accepted screening) and non-examinees (who declined screening).

\section{Results}

Only $16.5 \%$ of respondents were aware of the harms associated with thyroid cancer screening, with most perceiving that the benefits outweighed the harms. Comparison of subjects' and non-subjects' responses showed there were no significant differences between subjects and non-subjects. In addition, among subjects, there were no differences of responses between examinees and non-examinees. The most common reason for participation in screening was that the screening was conducted in schools and perceived as obligatory.

\section{Conclusions}

These results highlighted a serious ethical issue in that school-based screening leads to making young people think that it is mandatory screening in an opt-out and default setting manner, with a lack of knowledge about the disadvantages of screening. Based on the autonomy of the subjects and the ethical principle of the post-disaster, surveys after a nuclear disaster should be conducted in an opt-in style without an opt-out style such as school-based screening. 


\section{Background}

Screening for various diseases, including cancer, has both benefits and harms, such as overdiagnosis. Therefore, provision of adequate information is important in making decisions about whether to undergo screening. The harm associated with overdiagnosis is greater in children and adolescents than in adults, meaning that factors that aid decision making regarding screening for children and adolescents require more consideration [1]. However, after a nuclear accident, screening of radiation-related cancers, especially radioiodine-induced thyroid cancer, for residents may be conducted as part of a health survey. Media and social networks may amplify residents' radiation-related fears, and screening is often regarded as necessary for scientific and social reasons rather than concern about the harmful effects of screening $[2,3]$.

More than 10 years have passed since the Fukushima Daiichi Nuclear Power Plant accident that occurred immediately after the Great East Japan Earthquake and tsunami disaster in March 2011. It was difficult to communicate sufficiently with residents regarding the health risks associated with radiation exposure for some time after the Fukushima nuclear accident $[3,4]$. The level of radiation exposure among Fukushima residents was expected to be much lower than that among residents around Chernobyl after the 1986 accident [5]. However, based on experiences following the Chernobyl accident, residents in Fukushima were concerned about the increased risk for thyroid cancer among children and adolescents $[6,7]$. Thyroid ultrasound examination was started in Fukushima as part of the Fukushima Health Management Survey for all Fukushima residents (about 380,000 people) under age 18 years at the time of the accident [8]. This project started in October 2011, 6 months after the accident and intended to monitor children's health following the disaster. This timing meant that it was not possible to communicate with residents regarding the significance of the examination, including risks associated with radiation exposure in Fukushima, characteristics of juvenile thyroid cancer, and benefits and harms of thyroid cancer screening $[7,9]$. The thyroid ultrasound examination in Fukushima has been conducted as a screening at 2-year intervals since 2011 [8], but was initially conceptualized as a support program for residents who were worried about the health effects of radiation exposure [7]. However, there was a lack of communication of key information before the examination, especially regarding overdiagnosis. The screening program used an opt-out approach. All potential participants were notified about the venue and date of the examination, and examinations were also performed during school classes for school students [10].

A global perspective against thyroid cancer screening emerged during the 10 years in which the thyroid ultrasound examination has been conducted in Fukushima. For example, the rate of overdiagnosis following thyroid cancer screening in South Korea was described as "epidemic" and thyroid ultrasonography has been reported to increase the prevalence of thyroid cancer worldwide [11, 12] (Ahn et al. 2014; Vaccarella et al. 2016). Based on global reports, the US Preventive Services Task Force stated that thyroid cancer screening was not recommended for asymptomatic adults [13]. In addition, the International Agency for Research on Cancer (IARC) stated in 2018 that this screening was not recommended even after a nuclear accident [14]. A major reason for screening not being recommended is 
that evaluations suggested the harms of overdiagnosis by thyroid cancer screening outweighed the benefits.

However, four rounds of the thyroid ultrasound examination have been conducted in Fukushima over the past 10 years, and a fifth round with opt-out style screening is progressing despite these global trends. This examination has resulted in more than 200 thyroid cancer diagnoses (116 in the first round, 70 in the second, and 31 in the third) [15]. The United Nations Scientific Committee (UNSCEAR) concluded that the significant increase among screening participants relative to that expected was because of overdiagnosis and not the result of low radiation dose exposure [5]. There is concern that this examination will continue to cause issues related to overdiagnosis. However, once initiated, a screening program is difficult to cancel because of conflicts of interest and economic policies, such as with the mass screening of newborns for neuroblastoma in Japan [16] and thyroid cancer screening in South Korea [11]. The thyroid cancer screening program in Fukushima was also complicated because it started after the nuclear accident. As overdiagnosis is an emerging problem in recent health policy and practice [17], it is important to address its ethical implications. For example, it is necessary to clarify how well young people (as potential screening participants) understand the complex background and international changes in perspective regarding the harms of overdiagnosis following the thyroid ultrasound examination. The examination participation rate was high at $81.7 \%$ in the first round of screening, but showed a gradual decline to $71.0 \%$ in the second round and $61.3 \%$ in the third round [18]. However, detailed examination of the participation rate showed the participation rate of school students remained close to $90 \%$, even in the third round, whereas that in the age group after graduating from high school dropped sharply. This phenomenon may not occur if decision-making about receiving screening is based on anxiety about radiation health risks. It is therefore essential to understand how residents perceived the health risks of radiation exposure and harms of thyroid cancer screening when they decided whether to undergo screening. This information is important in reconsidering the thyroid ultrasound examination program in Fukushima as well as for future thyroid monitoring following a nuclear accident.

After a disaster, various research activities, including health surveys, tend to be conducted, but sometimes these activities are not beneficial for residents living in the affected areas $[19,20]$. It is an ethically important issue to determine why individuals make ongoing decisions to participate in potentially harmful examinations. To clarify factors that influenced the decision to undergo thyroid cancer screening in Fukushima, we conducted a questionnaire survey among young people in Fukushima, including those who received the thyroid examination and those who did not. In addition, we sent the questionnaire to young people of the same generation in a neighboring prefecture who were not potential thyroid examination participants as a comparative control.

\section{Methods}

We conducted an anonymous survey to clarify decision-making processes related to the thyroid ultrasound examination and associated ethical issues. The survey investigated three main areas. 
1. Reasons why the examinee took the thyroid ultrasound examination.

2. Perceptions of the significance, benefits, and harms of the thyroid ultrasound examination.

3. The impact of the examination being conducted during school classes.

\section{Respondents}

We sent an anonymous questionnaire to 2000 randomly selected young people (aged 16-20 and 25-30 years as at January 1,2020) who lived in Fukushima Prefecture (most of the subjects) and the neighboring Miyagi Prefecture (most of the non-subjects). Most residents these two prefectures were thought to have been affected by the Great East Japan Earthquake. The questionnaire was distributed via mail on January 7, 2020, and returned questionnaires with a postmark by February 15, 2020, were considered valid. Approval to conduct the survey was obtained from the Fukushima Medical University Ethics Committee (approval number: 2019-180). The questionnaire included a written explanation that returning a completed questionnaire would be considered provision of consent because the survey was completed anonymously and respondents were therefore not identifiable. Of the 601 young people (30.1\%) who responded, $594(29.7 \%)$ responses from those whose gender and age matched the population registry were considered valid. This questionnaire survey was planned and conducted while the authors were affiliated with Fukushima Medical University.

\section{Questionnaire content}

The questionnaire comprised five categories: 1) basic characteristics, 2) decision-making about participating in the thyroid ultrasound examination, 3) recognition of the benefits and harms of thyroid cancer screening, 4) risk perception relating to radiation-related health risks, and 5) the impact of conducting the examination during school classes.

The first category covered participants' age, sex, and a question asking whether they were the subject (all Fukushima residents under age 18 years at the time of the Fukushima accident).

The second category was only completed by those who were the subjects of the thyroid ultrasound examination in Fukushima. This category contained four questions. i) Do you know the meaning of the thyroid ultrasound examination? If you answer yes, please describe it. ii) Did you take the thyroid ultrasound examination in the last 2 years? iii) Who made the decision whether to take the examination? iv) Why did you take/not take the examination?

The third category asked all participants about: i) knowledge of benefits and harms of the thyroid ultrasound examination, ii) magnitude of benefits and harms of the thyroid ultrasound examination using a 5-point scale (more beneficial, beneficial, coequal, harmful, more harmful), and iii) knowledge about the IARC recommendation regarding thyroid cancer screening after a nuclear accident. For the analysis, 
"more beneficial" and "beneficial" were classified as "perceived beneficial" and "harmful" and "more harmful" were classified as "perceived harmful."

For the fourth category, we measured participants' risk perception of the potential health effects of radiation exposure based on their responses to two questions, with responses on a 4-point scale from very unlikely to very likely $[21,22]$. We investigated the possibility of delayed effects by asking, "What do you think is the likelihood of damage to your health (e.g., cancer onset) in later life as a result of your current level of radiation exposure?" The second question concerned the possibility of genetic effects: "What do you think is the likelihood that the health of your future (i.e., as-yet unborn) children and grandchildren will be affected as a result of your current level of radiation exposure?" For the analysis, "very unlikely" and "unlikely" were classified as lower risk perception and "likely" and "very likely" were classified as higher risk perception.

For the fifth category, we asked all participants to rate four statements regarding their perception of the impact of the examination conducted at school on a 5-point scale from likely to unlikely. The statements were: i) Examination at school (during classes) makes you perceive it as a good thing; ii) Examination at school makes you believe it is somewhat mandatory; iii) Examination at school makes it difficult to refuse taking the examination; and iv) The presence of people who were not attending the school examination made you feel as if there was something wrong.

\section{Analysis}

We conducted chi-square tests or non-parametric analyses for questions to compare risk perception, knowledge of harms of the examination, and basic characteristics between the subject and the nonsubject groups, and between those who received the examination (examinees) and those that did not (non-examinees). Reasons for receiving or not receiving the examination and the impact of school-based examination were explored using descriptive statistics.

\section{Results}

Table 1 presents a summary of the results of the comparisons of knowledge and perception between the examinees and non-examinees and between the subjects and non-subjects. There was no difference in the male to female ratio between the examinees and non-examinees or between subjects and nonsubjects. However, the mean age of the non-subjects was higher than that of the subjects, and the mean age of non-examinees was higher than that of examinees. This was because subjects were aged 18 years or younger at the time of the nuclear accident; therefore, the population aged 25-30 years who returned the questionnaire were all non-subjects, whereas the population aged 16-20 included both subjects and non-subjects.

The results showed that $40.5 \%$ of respondents who were subjects of the thyroid ultrasound examination did not know the meaning of the examination. In addition, there was no significant difference in 
knowledge between examinees and non-examinees. Furthermore, about half of those who knew the meaning of the examination provided incorrect descriptions; for example, "This examination measures the radiation level of the thyroid gland" (data not shown).

Regarding decision-making about taking the examination, $54.2 \%$ of examinees indicated the decision was made by their parents, and $15.3 \%$ made the decision themselves. In contrast, $57.0 \%$ of nonexaminees made the decision themselves, and $9.0 \%$ reported their parents made the decision. We believed that this corresponded to the decline in the examination participation rate after graduating from high school. Common reasons for taking the examination (multiple-choice question) were: 1) this was an examination done at school $(29.6 \%), 2$ ) concern about radiation effects $(13.5 \%)$, and 3 ) wanting to be relieved by undergoing the examination (11.1\%). Common reasons for not taking the examination were: 1) time-consuming (11.1\%) and 2 ) no worry about radiation exposure (11.1\%). However, no participant indicated "harms of the examination" was a reason for not receiving the examination.

Table 1 also shows that only $16.5 \%$ of respondents in the examinee and non-examinee groups knew about the harms of thyroid ultrasound examination. The proportion of those who knew about the harms was slightly smaller in the examinee group, but the difference between the groups was not significant. In addition, few people knew about the IARC recommendation against thyroid cancer screening, and there was no significant difference between groups. Furthermore, both examinees and non-examinees showed a tendency to overestimate the benefits compared with the harms of screening. The results regarding knowledge about the benefits and harms of the thyroid examination were similar for the non-subjects.

In terms of the risk perception of health effects caused by radiation exposure, there was no significant difference between examinees and non-examinees in responses to the questions concerning delayed and genetic effects. However, the risk perceptions for both delayed and genetic effects were significantly higher in non-subjects than in subjects (\% of higher delayed risk perception: $31.3 \%$ vs. $48.3 \%$; $\%$ of higher genetic risk perception: $21.2 \%$ vs. $36.8 \%$ ).

Figure 1 demonstrates the results of the analysis of items covering the impact of a school-based examination. In summary, these were:

i. About $80 \%$ of participants perceived the examination was good because it was a school-based examination.

ii. Around $78 \%$ of participants considered the school-based examination was somewhat mandatary.

iii. Overall, $70 \%$ of participants said that their preference not to take the examination was not respected.

iv. Finally, about half of the participants said they perceived something wrong about those who did not take the examination.

There were no differences in these opinions between the subject and non-subject groups, or between the examinee and non-examinee groups. 


\section{Discussion}

Because the risk for cancer due to radiation depends on the radiation dose, various activities to measure the radiation dose were conducted to respond to residents' health concerns after the Fukushima nuclear accident [23]. The radiation doses were expected to be extremely low in Fukushima [5], meaning that implementation of thyroid cancer screening did not logically correspond to the concerns about the health effects of radiation exposure. However, it has been noted that the high participation rate in the thyroid ultrasound examination in Fukushima may reflect the concerns about the health effects of radiation exposure among the parents of examination subjects, especially mothers $[7,9]$. In contrast, this study directly asked young people (who were the subjects) about the most common reason for taking the examination, which was because it was performed at school. This does not support the suggestion that many people took the examination to relieve anxiety about the health risk of thyroid cancer due to radiation exposure. This was also supported by our result that there was no significant difference in risk perception about radiation health effects (especially the risk for developing cancer such as delayed effects) between examinees and non-examinees. Furthermore, we found among those that took the examination, slightly more than half reported their parents made the decision to take the examination, whereas among those that did not take the examination, slightly more than half made this decision themselves. It has been reported that parents, especially mothers, were anxious about the increase in thyroid cancer after the Fukushima nuclear accident [6,7]. Therefore, parents' intentions may influence their decision to take the examination as well as the impact of the examination being conducted at school.

To support proper decision-making as to whether young people should take the screening examination, it is essential that they understand the significance, benefits, and harms of the examination. Our results showed that $40.5 \%$ of the thyroid ultrasound examination subjects did not know the meaning of the examination, and there was no significant difference in this knowledge between examinees and nonexaminees. In addition, the free-form answers showed that $47.8 \%$ of respondents who had this knowledge misunderstood the meaning of the examination (e.g., "This examination measures the radiation level of the thyroid gland"). This highlighted that the subjects, even examinees, of the thyroid ultrasound examination often did not understand the meaning of the examination correctly.

Most subjects were also unaware of the harms of thyroid cancer screening, and the percentage of those who knew there were harms associated with thyroid cancer screening was the same for examinees and non-examinees. It was also demonstrated that both examinees and non-examinees considered the benefits were greater than the harms. However, no respondents cited these harms as a reason for not taking the examination. These results suggested that potential thyroid examinees are somehow encouraged to undergo the examination in the decision-making process. Recently, it has been reported that examinees underestimate the disadvantages and overestimate the benefits of screening, testing, and intervention [24, 25]. It has also been noted that recognition of harms is important in decision making about breast cancer screening $[26,27]$. A qualitative study involving Korean women reported that many 
women were unaware of the potential harm of overdiagnosis associated with thyroid ultrasound screening [28].

We found that the risk perceptions of health effects due to radiation exposure were significantly higher in non-subjects than in subjects. This suggested that non-subjects (who were relatively far from the nuclear accident site) overestimated the radiation health risk compared with subjects. Similar results were shown in a survey by the National Institute of Advanced Industrial Science and Technology, where the risk perception of the general population in Tokyo was significantly higher than that of residents in the evacuation area in Fukushima [29]. These results indicated that the subjects had opportunities to access information and education on radiation health risks. In contrast, there was a non-significant difference in the awareness of the existence of harms of thyroid cancer screening between the subjects and nonsubjects. Moreover, there was overestimation of the benefits compared with the harms of thyroid cancer screening in both subjects and non-subjects. Subjects had received an explanation letter with guidance about taking the thyroid examination, whereas non-subjects did not receive a guidance letter. However, the benefit-harm perception did not differ between subjects and non-subjects. This suggested that the explanation and communication through the explanation letter before decision-making about taking the examination was insufficient.

This study showed that the examination at school led to the perception that the examination was a good thing or mandatory, and that preferences to not take the examination were not respected. Moreover, this perception was not specific to the subjects of the thyroid ultrasound examination, but was the same for non-subjects, which suggests that it is a common tendency among Japanese young people. Our data revealed that the methodology whereby the examination was conducted at school during classes could influence decision-making about taking the examination. This was supported by the sharp decline in the thyroid ultrasound examination participation rate after graduating from school [18]. These results indicated that the school-based examination became a "default," as an opt-out method was used. The impact of opt-in and opt-out methods on decision making has been reported in the issue of organ donation [30,31]. For example, the organ donation rate is high in countries with the opt-out condition, and low in countries with the opt-in condition. Even with major benefits of organ transplants, perspectives of the methodology of choosing opt-out or opt-in are divided. From an ethical perspective, care must be taken in adopting the opt-out method if a program has some potential harms. Because thyroid cancer screening has been shown to have major harms (e.g., overdiagnosis), it should not be performed in an opt-out and default-setting manner.

This study had several limitations. First, we identified the subjects/non-subjects and examinees/nonexaminees based on responses to category 1 . Based on the respondents' age and address, most of these responses were thought to be correct. Some respondents might have mistakenly answered based on another examination rather than the thyroid examination. Second, because the response rate in the present study was relatively low (30\%), the representativeness of the target populations was uncertain. However, the fact that a majority of respondents were unaware of the disadvantages of thyroid examination as a cancer screening tool is likely to be unaffected by bias of response rate. Third, because 
the age of non-subjects was higher that of the subjects, the risk perception of health effects due to radiation exposure might have been influenced by age. However, there was no report of a large difference in radiation health risk perception between the ages of $16-20$ and $25-30$ years.

\section{Conclusions}

We summarized the decision-making process and potential influencing factors for young subjects of the thyroid ultrasound examination in Fukushima in Figure 2. After a nuclear accident, residents are naturally concerned about the health effects of radiation exposure and tend to have high risk perception of radiation-related cancer. However, our results indicated there were no relationships in these factors. Since screening as a health survey tends to be conducted as a policy to resolve these concerns, opt-out style screening may result in misunderstanding that screening benefits outweighs the harms, especially school-based examinations. This is not limited to thyroid cancer screening, as screening to detect disease may not be beneficial for residents following a disaster $[10,19]$. To properly respond to anxieties of affected residents, it is essential to communicate key aspects to residents, including: 1) meaning of the examination, 2) balance between the benefits and harms of the examination, 3) characteristics of the target disease, and 4) voluntary participation. In addition, based on the principle of the post-disaster code of conduct, investigations and surveys after a disaster should be conducted in an opt-in style.

\section{Declarations}

\section{Ethics approval and consent to participate}

This study was approved by the Fukushima Medical University Ethics Committee, Fukushima, Japan (approval no. 2019-180). The questionnaire included an explanation that mailing a completed response was indicative of informed consent because the survey was completed anonymously and therefore no individuals were identifiable. All methods were performed in accordance with the Ethical Guidelines for Medical and Biological Research Involving Human Subjects by the Ministry of Education, Culture, Sports, Science and Technology of Japan.

\section{Consent for publication}

Not applicable.

\section{Availability of data and material}

Data other than those shown in tables, figures, and supplement are not publicly available.

\section{Conflicts of interest/Competing interests}

We declare no competing interests.

\section{Funding}


This work was partially supported by JSPS KAKENHI (Grant number 16K01791) and by the Fukushima Medical Association (Grant number KI24012).

\section{Authors' contributions}

Concept and design: Midorikawa and Ohtsuru. Analysis and drafting of the manuscript: Midorikawa. Critical revision of the manuscript: Ohtsuru.

\section{Acknowledgements}

We thank Dr. Michio Murakami and Dr. Yoshitake Takebayashi (Fukushima Medical University, Japan) for their academic support. We thank Ms. Mihoko Koizumi for her technical support (Fukushima Medical University, Japan). We also thank Audrey Holmes, MA, from Edanz (https://jp.edanz.com/ac) for editing a draft of this manuscript.

\section{References}

1. Coon ER, Quinonez RA, Moyer VA, Schroeder AR. Overdiagnosis: how our compulsion for diagnosis may be harming children. Pediatrics. 2014; doi: 10.1542/peds.2014-1778.

2. Normile D. Epidemic of fear. Science. 2016; doi: 10.1126/science.351.6277.1022

3. Ohtsuru A, Tanigawa K, Kumagai A, Niwa O, Takamura N, Midorikawa S et al. Nuclear disasters and health: lessons learned, challenges, and proposals. The Lancet 2015; doi: 10.1016/S01406736(15)60994-1.

4. Murakami M, Takebayashi Y, Takeda Y, Sato A, Igarashi Y, Sano K et al. Effect of radiological countermeasures on subjective well-being and radiation anxiety after the 2011 disaster: The Fukushima Health Management Survey. Int J Environ Res Public Health. 2018; doi: 10.3390/ijerph15010124.

5. UNSCEAR 2020 report. 2020. Sources, effects and risks of ionizing radiation. Annex B: Levels and effects of radiation exposure due to the accident at the Fukushima Daiichi Nuclear Power Station: implications of information published since the UNSCEAR 2013 Report. https://www.unscear.org/unscear/en/publications/2020b.html (accessed April 24, 2021).

6. Midorikawa S, Suzuki S, Ohtsuru A. After Fukushima: Addressing anxiety. Science. 2016; doi: 10.1126/science.352.6286.666-c.

7. Midorikawa S, Tanigawa K Suzuki S, Ohtsuru A. Psychosocial issues related to thyroid examination after a radiation disaster. Asia Pac J Public Health. 2017; doi: 10.1177/1010539516686164.

8. Suzuki S, Yamashita S, Fukushima T, Nakano K, Midorikawa S Ohtsuru S et al. The protocol and preliminary baseline survey results of the thyroid ultrasound examination in Fukushima [Rapid Communication]. Endoc J 2016; doi: 10.1507/endocrj.EJ15-0726

9. Hino Y, Murakami M, Midorikawa S, Ohtsuru A, Suzuki S, Tsuboi K, et al. Explanatory meetings on thyroid examination for the "Fukushima Health Management Survey" after the Great East Japan 
Earthquake: Reduction of anxiety and improvement of comprehension. Tohoku J Exp Med. 2016; doi: 10.1620/tjem.239.333.

10. Midorikawa S, Ohtsuru A. Disaster-zone research: make participation voluntary. Nature. 2020; doi: 10.1038/d41586-020-00695-0.

11. Ahn HS, Kim HJ, Welch HG. Korea's thyroid-cancer "epidemic" --screening and overdiagnosis. N Engl J Med. 2014; doi: 10.1056/NEJMp1409841.

12. Vaccarella S, Franceschi S, Bray F, Wild CP, Plummer M, Dal Maso L. Worldwide thyroid cancer epidemic? The increasing impact of overdiagnosis. N Engl J Med. 2016; doi:

10.1056/NEJMp1604412

13. US Preventive Services Task Force, Bibbins-Domingo K, Grossman DC, Curry SJ, Barry MJ, Davidson KW, et al. Screening for thyroid cancer: US Preventive Services Task Force Recommendation Statement. JAMA. 2017; doi: 10.1001/jama.2017.4011.

14. Togawa K, Ahn HS, Auvinen A, Bauer AJ, Brito JP, Davies L et al. Long-term strategies for thyroid health monitoring after nuclear accidents: recommendations from an expert group convened by IARC. Lancet Oncol. 2018; doi: 10.1016/S1470-2045(18)30680-6

15. Ohtsuru, A, Midorikawa S. Lessons learned from conducting disease monitoring in low-dose exposure conditions as a counter-measure after a nuclear disaster. J Radiat Res. 2021; doi: 10.1093/jrr/rraa105.

16. Tsubono Y Hisamichi S. A halt to neuroblastoma screening in Japan. N Engl J Med. 2004; doi: 10.1056/NEJM200405063501922

17. Takano T. Overdiagnosis of thyroid cancer: The children in Fukushima are in danger. Arch Pathol Lab Med. 2019; doi: 10.5858/arpa.2018-0586-LE

18. th Fukushima Prefecture Thyroid Examination Evaluation Subcommittee, June 15, 2020. https://www.pref.fukushima.lg.jp/site/portal/kenkocyosa-kentoiinkai-b15.html (in Japanese). Accessed 16 June 2021.

19. Gaillard JC, Peek L. Disaster-zone research needs a code of conduct. Nature. 2019; doi: 10.1038/d41586-019-03534-z.

20. Oughton D, Liutsko L, Midorikawa S, Pirard P, Schneider T, Tomkiv Y. An etical dimension to accident management and health surveillance. Environ Int. 2021: doi: 10.1016/j.envint.2021.106537

21. Lindell, MK, Barnes VE. Protective response to technological emergency: risk perception and behavioral intention. Nuclear Safety. 1986; doi: 10.1016/0022-4375(89)90021-2.

22. Suzuki Y, Yabe H, Yasumura S, Ohira O, Niwa S, Ohtsuru A et al. Psychological distress and the perception of radiation risks in Fukushima evacuees. Bull World Health Organ. 2015; doi: 10.2471/BLT.14.146498

23. Murakami M, Kumagai A, Ohtsuru A. Building risk communication capabilities among professionals: seven essential characteristics of risk communication. Radiat Prot Dosimetry. 2018; doi: 10.1093/rpd/ncy 140 . 
24. Hoffmann TC, Del Mar C. Patients' expectations of the benefits and harms of treatments, screening, and tests: a systematic review. JAMA Intern Med. 2015; doi: 10.1001/jamainternmed.2014.6016.

25. Hoffmann TC, Del Mar C. Clinicians' expectations of the benefits and harms of treatments, screening, and tests: A systematic review. JAMA Intern Med. 2017; doi: 10.1001/jamainternmed.2016.8254.

26. Attena, F, Cancellieri M, Pelullo CP. Scarce information about breast cancer screening: An Italian websites analysis. Medicine (Baltimore). 2016; doi: 10.1097/MD.0000000000005615.

27. Independent UK Panel on Breast Cancer Screening. The benefits and harms of breast cancer screening: an independent review. The Lancet. 2012; doi: 10.1016/S0140-6736(12)61611-0.

28. Park SH, Lee B, Lee S, Choi E, Choi EB, Yoo J, Jun JK, Choi KS. A qualitative study of women's views on overdiagnosis and screening for thyroid cancer in Korea. BMC Cancer. 2015; doi: $10.1186 /$ s12885-015-1877-6

29. Yoshizawa N, Shirai K, Murakami K Baba T. Fukushima Reconstruction: Current Status and Radiation Health Risks. 2017. https://www.mri.co.jp/knowledge/column/i6sdu6000000u86patt/MTR_Fukushima_1712.pdf Accessed 16 June 2021.

30. Johnson EJ, Goldstein D. Do defaults save lives? Science. 2003; doi: 10.1126/science.1091721

31. Davidai S, Gilovich T, Ross LD. The meaning of default options for potential organ donors. Proc Natl Acad Sci U S A. 2012; doi: 10.1073/pnas.1211695109.

\section{Tables}

Due to technical limitations, table 1 is only available as a download in the Supplemental Files section.

\section{Figures}




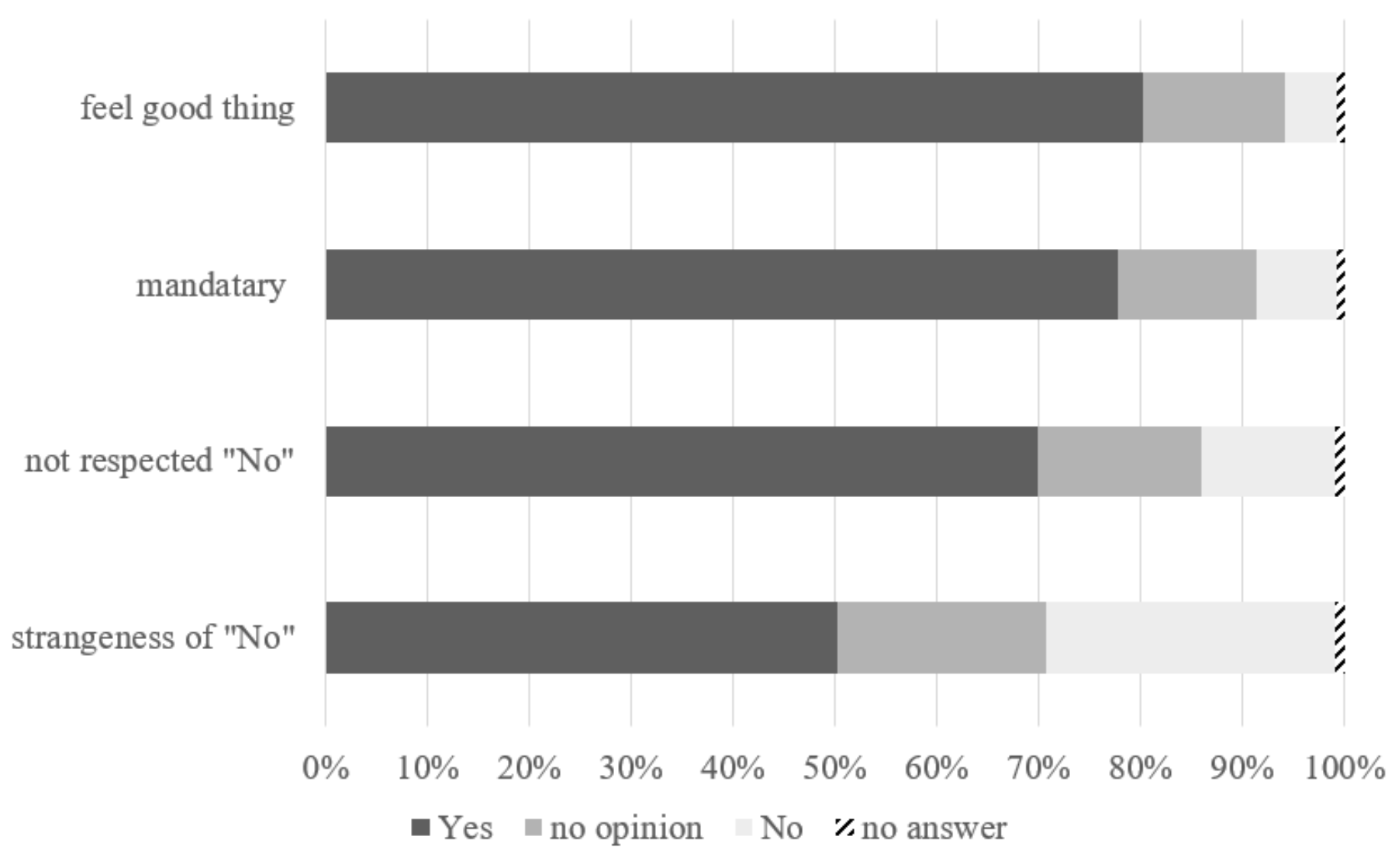

\section{Figure 1}

Impact of the school-based thyroid examination The percentage of "Yes" responses are shown for the following items related to the recognition of a school-based examination. i) $80 \%$ of participants perceived the examination was good thing. ii) $78 \%$ of participants considered the school-based examination was somewhat mandatary. iii) $70 \%$ of participants said that their preference not to take the examination was not respected. iv) About half of the participants felt there was something wrong about those who did not take the examination. The remaining percentages for each item show responses of "No," "no opinion," or "no answer." 


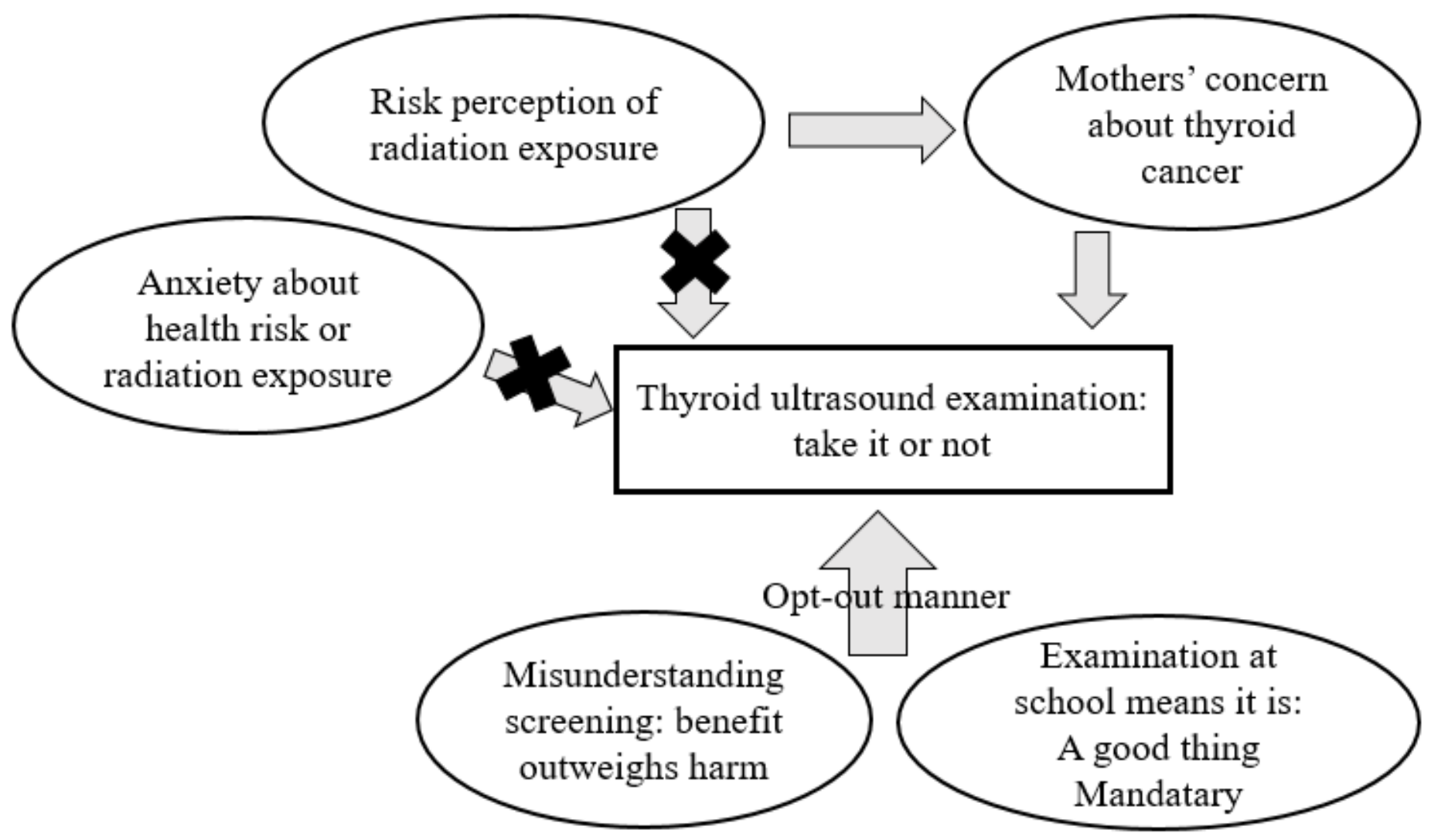

Figure 2

Decision-making process and potential influencing factors for young subjects of the thyroid ultrasound examination in Fukushima There were several potential factors that influenced whether the subjects took the thyroid ultrasound examination after the nuclear accident.

\section{Supplementary Files}

This is a list of supplementary files associated with this preprint. Click to download.

- Table1.pptx

- aditionalfilequestionnaire.docx 